\title{
Speech Recognition by Wavelet Analysis
}

\author{
Nitin Trivedi \\ Asstt. Prof. \\ Vidya College of Engg. \\ Meerut \\ Sachin Ahuja \\ Asstt. Prof. \\ Vidya College of Engg. \\ Meerut
}

\author{
Dr. Vikesh Kumar \\ Director \\ Vidya College of Engg. \\ Meerut
}

\author{
Raman Chadha \\ Asstt. Prof. \\ Vidya College of Engg. \\ Meerut
}

\author{
Saurabh Singh \\ Asstt. Prof. \\ Vidya College of Engg. \\ Meerut
}

\begin{abstract}
In an effort to provide a more efficient representation of the speech signal, the application of the wavelet analysis is considered. This research presents an effective and robust method for extracting features for speech processing. Based on the time-frequency multi-resolution property of wavelet transform, the input speech signal is decomposed into various frequency channels.

The major issues concerning the design of this Wavelet based speech recognition system are choosing optimal wavelets for speech signals, decomposition level in the DWT, selecting the feature vectors from the wavelet coefficients. More specifically automatic classification of various speech signals using the DWT is described and compared using different wavelets. Finally, wavelet based feature extraction system and its performance on an isolated word recognition problem are investigated. For the classification of the words, three layered feed forward network is used.
\end{abstract}

\section{General Terms}

Dynamic Time Warping (DTW) Algorithm, Wavelet Transform (WT).

\section{Keywords}

Speech recognition, feature extraction, wavelet transform, Discrete Wavelet Transform (DWT).

\section{INTRODUCTION}

Speech recognition is the process of automatically extracting and determining linguistic information conveyed by a speech signal using computers or electronic circuits. Automatic speech recognition methods, investigated for many years have been principally aimed at realizing transcription and human computer interaction systems. The first technical paper to appear on speech recognition has since then intensified the researches in this field, and speech recognizers for communicating with machines through speech have recently been constructed, although they remain only of limited use.

Automatic speech recognition (ASR) features some of the following advantages:

- Speech input is easy to perform because it does not require a specialized skill as does typing or pushbutton operations.

- Information can be input even when the user is moving or doing other activities involving the hands, legs, eyes, or ears.

- Since a microphone or telephone can be used as an input terminal, inputting information is economical with remote inputting capable of being accomplished over existing telephone networks and the Internet.

However, the task of ASR is difficult because:

- Lot of redundancy is present in the speech signal that makes discriminating between the classes difficult.

- Presence of temporal and frequency variability such as intra speaker variability in pronunciation of words and phonemes as well as inter speaker variability e.g. the effect of regional dialects.

- Context dependent pronunciation of the phonemes (co-articulation).

- Signal degradation due to additive and convolution noise present in the background or in the channel

- Signal distortion due to non-ideal channel characteristic.

\section{SPEECH RECOGNITION}

Most speech recognition systems can be classified according to the following categories:

\subsection{Speaker Dependent vs. Speaker Independent}

A speaker-dependent speech recognition system is one that is trained to recognize the speech of only one speaker. Such systems are custom built for just a single person, and are hence not commercially viable. Conversely, a speaker-independent system is one that is independence is hard to achieve, as speech recognition systems tend to become attuned to the speakers they are trained on, resulting in error rates that are higher than speaker dependent systems.

\subsection{Isolated vs. Continuous}

In isolated speech, the speaker pauses momentarily between every word, while in continuous speech the speaker speaks in a continuous and possibly long stream, with little or no breaks in between. Isolated speech recognition systems are easy to build, as it is trivial to determine where one word ends and another starts, and each word tends to be more cleanly and clearly spoken. Words spoken in continuous speech on the other hand are subjected to the co-articulation effect, in which the pronunciation of a word is modified by the words surrounding it. This makes training a speech system difficult, as there may be many inconsistent pronunciations for the same word.

2.3 Keyword-based vs. Sub-word unit based A speech recognition system can be trained to recognized whole words, like dog or cat. This is useful in applications 
like voice-command-systems, in which the system need only recognize a small set of words. This approach, while simple, is unfortunately not scalable [8]. As the dictionary of recognized words grow, so too the complexity and execution time of the recognizer. A more practical approach would be to train the recognition system to recognize sub-word units like syllables or phonemes (phonemes are the smallest atomic speech sound, like the ' $w$ ' and 'iy' sounds in 'we'), and then re-construct the word based on which syllables or phonemes are recognized.

For speech recognition, some of its characteristics (features) in time/frequency or in some other domain must be known. So a basic requirement of a speech recognition system will be to extract a set of features for each of the basic units. A feature can be defined as a minimal unit, which distinguishes maximally close units. The feature vector extracted should possess the following properties:

- Vary widely from class to class.

- Stable over a long period of time.

- Can be easily computed from the input speech samples.

- Should be small in dimension.

- Should be insensitive to the irrelevant variation in the speech.

- Should not have correlation with other features.

\section{WAVELET ANALYSIS}

\subsection{Introducing Wavelet}

The fundamental idea behind wavelets is to analyze according to scale. The wavelet analysis procedure is to adopt a wavelet prototype function called an analyzing wavelet or mother wavelet. Any speech signal can then be represented by translated and scaled versions of the mother wavelet.

Wavelet analysis is capable of revealing aspects of data that other speech signal analysis technique such the extracted features are then passed to a classifier for the recognition of isolated words.

\subsection{Statement of the Problem}

In this research, the problem of recognizing a small set of prescribed vocabulary words spoken is investigated. It describes a new method for speaker-independent word recognition using wavelet transform (WT) features. While much research has been performed in cepstral analysis, very little has been performed in wavelet domain for speech analysis. In principle, this research is a modification of the previous methods which is applied for speech recognition. The differences between the present word recognition method and the previous method lie in the features selected for analysis and in the length of the period for extracting the wavelet features. The number of levels of wavelet decomposition and the type of decomposition are different from the previous methods applied for speech recognition. Lastly, some of the wavelet features are proposed which are new in speech recognition.

\subsection{Examples of Wavelet}

The different families make trade-offs between how compactly the basis functions are localized in space and how smooth they are. Within each family of wavelets (such as the Daubechies family) are wavelet subclasses distinguished by the number of filter coefficients and the level of iteration. Wavelets are most often classified within a family by the number of vanishing moments. This is an extra set of mathematical relationships for the coefficients that must be satisfied. The extent of compactness of signals depends on the number of vanishing moments of the wavelet function used.

\subsection{Daubechies-N Wavelet family}

The Daubechies wavelet is one of the popular wavelets and has been used for speech recognition [1]. It is named after its inventor, the mathematician Ingrid Daubechies. These wavelets have no explicit expression except for db1, which is the Haar wavelet. The Daubechies wavelets properties:

- The support length of the wavelet function $\Psi$ and the scaling function $\Phi$ is $2 \mathrm{~N}-1$. The number of vanishing moments of $\Psi$ is $\mathrm{N}$.

- Most dbN are not symmetrical.

- The regularity increases with the order. When $\mathrm{N}$ becomes very large, $\Psi$ and $\Phi$ belong to $C^{\mu \mathrm{N}}$ where $\mu$ is approximately equal to 0.2 .

Daubechies-8 wavelet is used for decomposition of speech signal as it needs minimum support size for the given number of vanishing points [4].

\section{THE DISCRETE WAVELET TRANSFORM}

The Discrete Wavelet Transform (DWT) involves choosing scales and positions based on powers of two - so called dyadic scales and positions [5].

The mother wavelet is rescaled or dilated, by powers of two and translated by integers.

Specifically, a function $f(t) \in L^{2}(R)$ (defines space of square integrable functions) can be represented as $\mathrm{f}(\mathrm{t})=\sum_{\mathrm{J}=1-\mathrm{L}} \sum_{\mathrm{K}=\mathrm{a}}$ to $-\alpha={ }^{-j}(\mathrm{j}, \mathrm{k}) \psi\left(2^{-\mathrm{j}} \mathrm{t}-\mathrm{k}\right)+\Sigma_{\mathrm{K}=\alpha \text { to }-\alpha} \mathrm{a}(\mathrm{L}, \mathrm{k}) \varphi\left(2^{-\mathrm{L}} \mathrm{t}-\mathrm{k}\right)$

The function $\psi(\mathrm{t})$ is known as the mother wavelet, while $\varphi(\mathrm{t})$ is known as the scaling function. The set of functions $\{\sqrt{ } 2$ $\left.\varphi\left(2^{-\mathrm{L}} \mathrm{t}-\mathrm{k}\right), \sqrt{ } 2^{-\mathrm{j}} \psi\left(2^{-\mathrm{j}} \mathrm{t}-\mathrm{k}\right) \mid \mathrm{j}<=\mathrm{L}, \mathrm{j}, \mathrm{k}, \mathrm{LEZ}\right\}$, where $\mathrm{Z}$ is the set of integers, is an orthonormal basis for $\mathrm{L}(\mathrm{R})$.

The numbers $\mathrm{a}(\mathrm{L}, \mathrm{k})$ are known as the approximation coefficients at scale $\mathrm{L}$, while $\mathrm{d}(\mathrm{j}, \mathrm{k})$ are known as the detail coefficients at scale $\mathrm{j}$. The approximation and detail coefficients can be expressed as:

$$
\begin{aligned}
& \mathrm{a}(\mathrm{L}, \mathrm{k})=1 / \sqrt{ } 2 \int_{\alpha}^{-\alpha} \mathrm{f}(\mathrm{t}) \varphi\left(2^{-\mathrm{L}} \mathrm{t}-\mathrm{k}\right) \mathrm{dt} \\
& \mathrm{d}(\mathrm{j}, \mathrm{k})=1 / \sqrt{ } 2 \int_{\alpha}^{-\alpha} \mathrm{f}(\mathrm{t}) \psi\left(2^{-\mathrm{j}} \mathrm{t}-\mathrm{k}\right) \mathrm{dt}
\end{aligned}
$$

To provide some understanding of the above coefficients consider a projection $\mathrm{f}_{1}(\mathrm{t})$ of the function $\mathrm{f}(\mathrm{t})$ that provides the best approximation (in the sense of minimum error energy) to $\mathrm{f}(\mathrm{t})$ at a scale 1 . This projection can be constructed from the coefficients a(L,k), using the equation

$$
\mathrm{f}(\mathrm{t})=\Sigma_{\mathrm{K}=\alpha \text { to }-\alpha} \mathrm{a}(\mathrm{l}, \mathrm{k}) \varphi\left(2^{-1} \mathrm{t}-\mathrm{k}\right)
$$

As the scale 1 decreases, the approximation becomes finer, converging to $f(t)$ as $l \rightarrow 0$. The difference between the approximation at scale $1+1$ and that at $1, f_{1+1}(t)-f_{1}(t)$, is completely described by the coefficients $d(j, k)$ using the equation

$$
\mathrm{f}_{\mathrm{l}+1}(\mathrm{t})-\mathrm{f}_{1}(\mathrm{t})=\Sigma_{\mathrm{K}=\alpha \text { to }-\alpha} \mathrm{d}(1, \mathrm{k}) \psi\left(2^{-1} \mathrm{t}-\mathrm{k}\right)
$$

Using these relations, given $a(L, k)$ and $\{d(j, k) \mid j<=L\}$, it is clear that we can build the approximation at any scale. Hence, the wavelet transform breaks the signal up into a coarse approximation $\mathrm{f}_{\mathrm{L}}(\mathrm{t})$ (given $\mathrm{a}(\mathrm{L}, \mathrm{k})$ ) and a number of layers of detail $\left\{\mathrm{f}_{\mathrm{j}+1}(\mathrm{t})-\mathrm{f}_{\mathrm{j}}(\mathrm{t}) \mid \mathrm{j}<\mathrm{L}\right\} \quad$ (given by $\{\mathrm{d}(\mathrm{j}, \mathrm{k}) \mid \mathrm{j} \leq \mathrm{L}\}$ ). As each layer of detail is added, the approximation at the next finer scale is achieved. 


\subsection{The Fast Wavelet Transform Algorithm} The Discrete Wavelet Transform (DWT) coefficients can be computed by using Mallat's [2] Fast Wavelet Transform algorithm. This algorithm is sometimes referred to as the two-channel sub-band coder and involves filtering the input signal based on the wavelet function used. Consider the following equations:

$$
\begin{aligned}
& \varphi(\mathrm{t})=\sum_{\mathrm{k}} \mathrm{c}(\mathrm{k}) \varphi(2 \mathrm{t}-\mathrm{k}) \\
& \psi(\mathrm{t})=\sum_{\mathrm{K}}(-1) \mathrm{k}(1-\mathrm{k}) \varphi(2 \mathrm{t}-\mathrm{k}) \\
& \sum_{\mathrm{K}}^{\mathrm{c}} \mathrm{c}_{\mathrm{k}-2 \mathrm{~m}}^{\mathrm{m}}=2 \delta_{0, \mathrm{~m}}
\end{aligned}
$$

The first equation is known as the twin-scale relation (or the dilation equation) and defines the scaling function $\varphi$. The next equation expresses the wavelet $\psi$ in terms of the scaling function $\varphi$. The third equation is the condition required for the wavelet to be orthogonal to the scaling function.

The coefficients $\mathrm{c}(\mathrm{k})$ or $\left\{\mathrm{c}_{0}, \ldots . ., \mathrm{c}_{2 \mathrm{~N}-1}\right\}$ in the above equations represent the impulse response coefficients for a low pass filter of length $2 \mathrm{~N}$, with a sum of 1 and a norm of $1 / \sqrt{ } 2$.

Starting with a discrete input signal vector $\mathrm{s}$, the first stage of the FWT algorithm decomposes the signal into two sets of coefficients. These are the approximation coefficients $\mathrm{cA}$ (low frequency information) and the detail coefficients $\mathrm{cD}$ (high frequency information), as shown in the figure below.

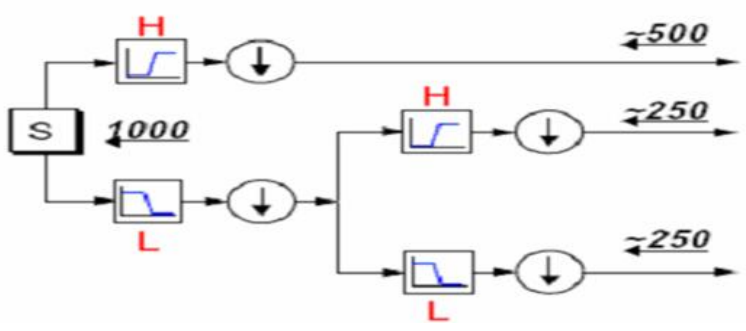

Fig 1: Filtering Analysis of DWT

\subsection{Multilevel Decomposition}

The decomposition process can be iterated, with successive approximations being decomposed in turn, so that one signal is broken down into many lower resolution components. This is called the wavelet decomposition tree [6].

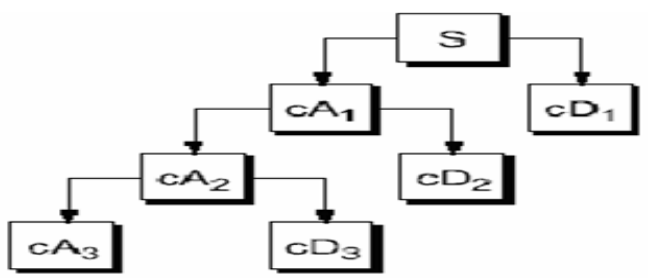

Fig 2: Decomposition of DWT Coefficient

The wavelet decomposition of the signal's' analyzed at level ' $\mathrm{j}$ ' has the following structure $\left[\mathrm{cA}_{\mathrm{j}}, \mathrm{cD}_{j}, \ldots, \mathrm{cD}_{1}\right]$.

Looking at a signals wavelet decomposition tree can reveal valuable information. The diagram below shows the wavelet decomposition to level 3 of a sample signal $\mathrm{S}$.

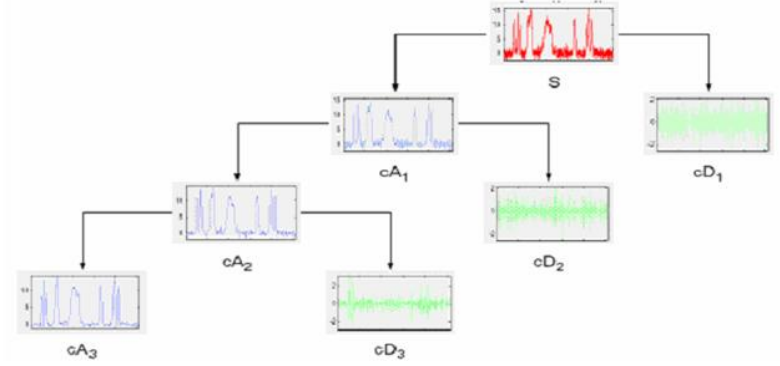

Fig 3: Level 3 Decomposition of Sample Signal S

Since the analysis process is iterative, in theory it can be continued indefinitely. In reality, the decomposition can only proceed until the vector consists of a single sample. Normally, however there is little or no advantage gained in decomposing a signal beyond a certain level. The selection of the optimal decomposition level in the hierarchy depends on the nature of the signal being analyzed or some other suitable criterion, such as the low-pass filter cut-off.

\subsection{Signal Reconstruction}

The original signal can be reconstructed or synthesized using the inverse discrete wavelet transform (IDWT).

The synthesis starts with the approximation and detail coefficients $\mathrm{cA}_{j}$ and $\mathrm{cD}_{j}$, and then reconstructs $\mathrm{cA}_{j-1}$ by up sampling and filtering with the reconstruction filters.

The reconstruction filters are designed in such a way to cancel out the effects of aliasing introduced in the wavelet decomposition phase [8]. The reconstruction filters (Lo_R and Hi_R) together with the low and high pass decomposition filters, forms a system known as quadrature mirror filters (QMF).

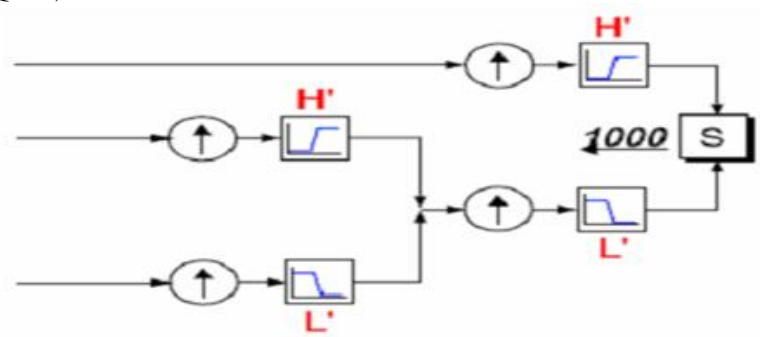

Fig 4: Signal Reconstruction

\section{METHODOLOGY}

The different steps involved in the proposed method are preprocessing, frame blocking \& windowing, wavelet feature extraction and the word recognition module which are given as follows:

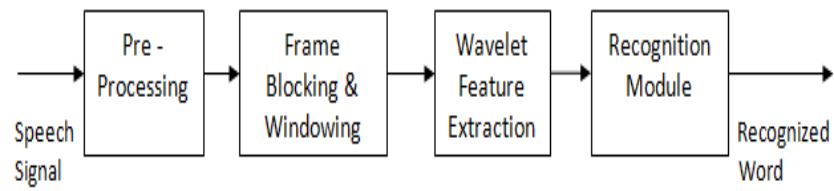

Fig 5: Design overview of speech recognition process

\subsection{Preprocessing}

The objective in the preprocessing is to modify the speech signal, so that it will be more suitable for the feature extraction analysis. The preprocessing consists of de-noising, pre-emphasis and voice activation detection. 


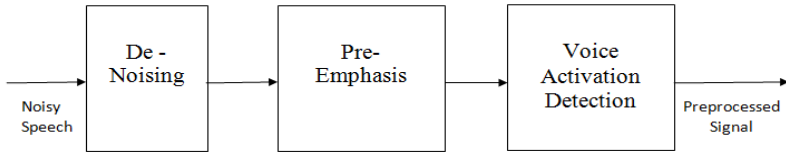

Fig 6: Preprocessing of Speech Signal

Automatic speech recognition involves a number of disciplines such as physiology, acoustics, signal processing, pattern recognition, and linguistics. The difficulty of automatic speech recognition is coming from many aspects of these areas. A survey of robustness issues in automatic speech recognition may be found in [3]

\subsection{Voice Activation Detection (VAD)}

The problem of locating the endpoints of an utterance in a speech signal is a major problem for the speech recognizer. Inaccurate endpoint detection will decrease the performance of the speech recognizer. The problem of detecting endpoints seems to be relatively trivial, but it has been found to be very difficult in practice. Some commonly used measurements for finding speech are short-term energy estimate $\mathrm{E}_{\mathrm{sl}}$, or short-term power estimate $\mathrm{P}_{\mathrm{sl}}$, and short term zero crossing rate $\mathrm{Z}_{\mathrm{s} 1}$. For the speech signal $\mathrm{s}_{1}(\mathrm{n})$ these measures are calculated as follows:

$$
\begin{aligned}
& \mathrm{E}_{\mathrm{s} 1}(\mathrm{~m})=\sum_{\mathrm{n}=\mathrm{m}-\mathrm{L}+1 \text { to } \mathrm{m}} \mathrm{S}_{1}^{2}(\mathrm{n}) \\
& \mathrm{P}_{\mathrm{s} 1}(\mathrm{~m})=1 / \mathrm{L} \sum_{\mathrm{n}=\mathrm{m}-\mathrm{L}+1 \text { to } \mathrm{m} 1} \mathrm{~s} \text { (n) } \\
& \mathrm{Z}_{\mathrm{s} 1}(\mathrm{~m})=1 / \mathrm{L} \sum_{\mathrm{n}=\mathrm{m}-\mathrm{L}+1} \text { to } \operatorname{m}_{1}^{\mid \operatorname{sgn}(\mathrm{s}(\mathrm{n}))} \\
& \operatorname{sgn}(\mathrm{s}(\mathrm{n}-1)) \mid / 2 \\
& \begin{array}{c}
\operatorname{sgn}\left(s_{1}(n)\right)=+1 \text { if } s_{1}(n)>0 \\
1 \\
\operatorname{sgn}\left(s_{1}(n)\right)=-1 \text { if } s_{1}(n) \leq 0
\end{array}
\end{aligned}
$$

Where $\quad \operatorname{sgn}(\mathrm{s}(\mathrm{n}))=+1$ if $\mathrm{s}(\mathrm{n})>0$

These measures will need some triggers for making decision about where the utterances begin and end. To create a trigger, one should need some information about the background noise. This is done by assuming that the first 10 blocks are background noise. The trigger for this function can be described as:

$$
\mathrm{t}_{\mathrm{w}}=\mu_{\mathrm{w}}+\alpha \delta_{\mathrm{w}}
$$

The $\mu_{w}$ is the mean and $\delta_{w}$ is the variance calculated for the first 10 blocks. The $\alpha$ term is a constant that have to be fine

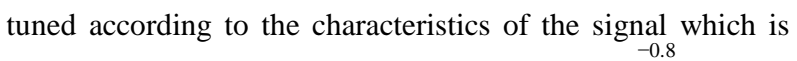
given as

$$
\alpha=0.2 \delta
$$

The voice activation detection function, $\operatorname{VAD}(\mathrm{m})$, can now be found as:

$$
\begin{aligned}
& \operatorname{VAD}(\mathrm{m})=1, \\
& \mathrm{~W}(\mathrm{~m}) \geq \mathrm{t}=0, \mathrm{~W}(\mathrm{~m})<\mathrm{t} \\
& \text { Where } \mathrm{W}_{\mathrm{s} 1}(\mathrm{~m})=\mathrm{P}_{\mathrm{s} 1}(\mathrm{~m}) \cdot\left(1-\mathrm{Z}_{\mathrm{s} 1}(\mathrm{~m})\right) \cdot \mathrm{S}_{\mathrm{c}} \mathrm{S}_{\mathrm{c}}=1000
\end{aligned}
$$

\subsection{Frame Blocking and Windowing}

Before Frame blocking and Windowing, the duration of the word must be equal for all the utterances in order to divide the signal into equal number of frames. This can be achieved by using Dynamic Time Warping (DTW) explained below:

\section{Dynamic Time Warping}

Even if the same speaker utters the same word, the duration changes every time with nonlinear expansion and contraction. Therefore, Dynamic Time Warping (DTW) is essential at the word recognition stage. The DTW process nonlinearly expands or contracts the time axis to match the same word.

The DTW algorithm is given as follow:

Let $\mathrm{A} \& \mathrm{~B}$ be the sequence vectors which are to be compared, $\mathrm{A}=\mathrm{a}_{1}, \mathrm{a}_{2}, \mathrm{a}_{3}, \ldots \ldots \ldots, \mathrm{a}_{\mathrm{I}}$ and $\mathrm{B}=\mathrm{b}_{1}, \mathrm{~b}_{2}, \mathrm{~b}_{3}, \ldots \ldots, \mathrm{b}_{\mathrm{J}}$

The warping function indicating the correspondence between the time axes of $\mathrm{A}$ and $\mathrm{B}$ sequences can be represented by a sequence of lattice points on the plane, $\mathrm{c}=(\mathrm{i}, \mathrm{j})$, as $\mathrm{F}=\mathrm{c}_{1}, \mathrm{c}_{2}, \mathrm{c}_{3} \ldots \mathrm{c}_{\mathrm{k}, \mathrm{k}}^{\mathrm{c}}=(\mathrm{i}, \mathrm{j})$

When the spectral distance between two feature vectors a and b is presented by $d(c)=d(i, j)$, the sum of the distances from beginning to end of the sequences along $F$ can be represented by

$$
\mathrm{D}(\mathrm{F})=\sum_{\mathrm{k}=1 \text { to } \mathrm{K}} \mathrm{d}\left(\mathrm{c}_{\mathrm{k}}\right) \mathrm{w}_{\mathrm{k}} / \sum_{\mathrm{k}=1 \text { to K }} \mathrm{w}_{\mathrm{k}}
$$

The smaller the value of $\mathrm{D}(\mathrm{F})$, the better the match between $\mathrm{A}$ and $\mathrm{B} . \mathrm{w}_{\mathrm{k}}$ is a positive weighting function related to $\mathrm{F}$.

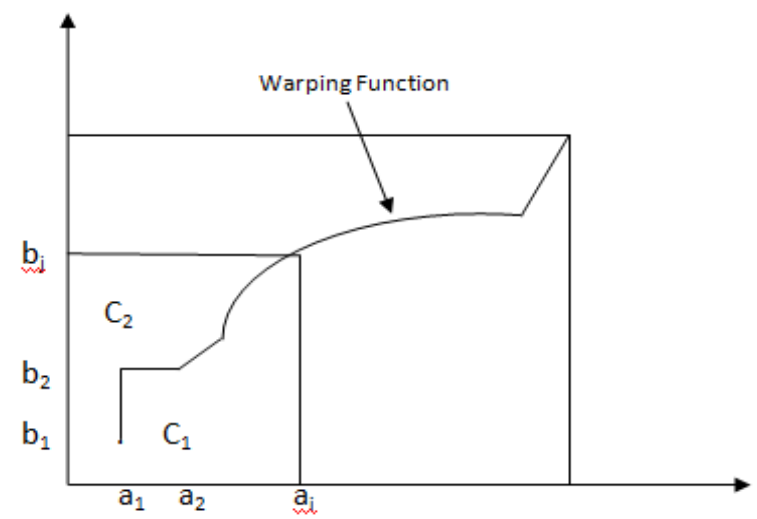

Fig 7: Dynamic Time warping between two time sequences

For minimizing the concerning $\mathrm{F}$ consider the following conditions.

1. Monotony and continuity condition

$$
0 \leq \mathrm{i}_{\mathrm{k}}-\mathrm{i}_{\mathrm{k}-1} \leq 1,0 \leq \mathrm{j}_{\mathrm{k}}-\mathrm{j}_{\mathrm{k}-1} \leq 1
$$

2. Boundary condition $\mathrm{i}_{1}=\mathrm{j}_{1}=1, \mathrm{i}_{\mathrm{k}}=\mathrm{I}, \mathrm{j}_{\mathrm{K}}=\mathrm{J}$

3. Adjustment window condition

$$
\begin{aligned}
& \left.\left|i_{k}-i_{k}\right| \leq r, r=\text { constant if } w_{k}=\left(i_{k}-i_{k-1}\right)+j_{k}-j_{k-1}\right) \text { then } \\
& \sum_{k=1} \text { to } K w_{k}=I+J \text {. Also } \\
& D(F)=1 /(I+J) \Sigma_{k=1 \text { to } K} d(c) w_{k} .
\end{aligned}
$$

Since, the objective function to be minimized becomes additive, minimization can be efficiently done as follows:

$$
g\left(C_{k}\right)=g(i, j)=\min c_{k-1}\left[g\left(c_{k-1}\right)+d\left(c_{k}\right) w_{k}\right]
$$

Which can be rewritten as

$$
\begin{aligned}
& g(i, j)=\min \{g(1, j-1)+d(i, j), g(1-i, j-1)+ \\
& 2 d(i, j), g(1-i, j)+d(i, j)\} \\
& g(1,1)=2 d(1,1)
\end{aligned}
$$

Once Dynamic Time Warping is done, the next step is to divide the speech signal into different frames and then applying hamming windowing for each frame which can be done as follows:

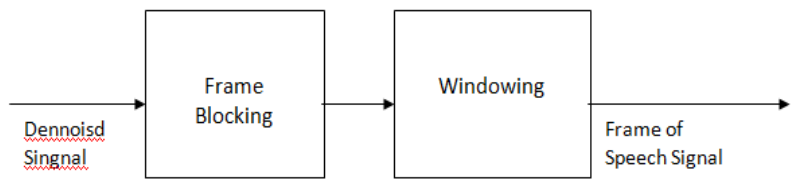

Fig 8: Frame blocking \& windowing 
For each utterances of the word, a window duration of $\mathrm{T}=32 \mathrm{~ms}$ is used for processing at later stages. A frame is formed from the windowed data with typical frame duration (Tf) of about $20 \mathrm{~ms}$. Since the frame duration is shorter than window duration there is an overlap of data and the percentage overlap is given as:

\section{$\%$ Overlap $=\left(\left(\mathrm{T}_{\mathrm{w}}-\mathrm{T}\right) * 100\right) / \mathrm{T}$}

Each frame is $32 \mathrm{~ms}$ samples long, with adjacent frames being separated by $20 \mathrm{~ms}$ samples as shown in the following diagram.

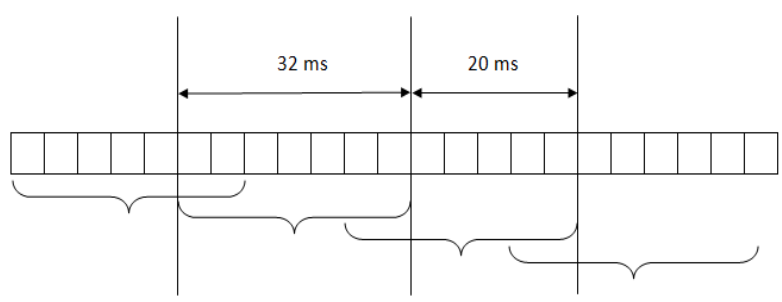

Fig 9: Frame blocking of a sequence

The Hamming window to each frame is applied in order to reduce signal discontinuity at either end of the block. It is calculated as follows:

$$
\mathrm{w}(\mathrm{k})=0.54-0.46 \cos (2 \Pi \mathrm{k} / \mathrm{K}-1)
$$

\section{Result}

The results obtained after each stage are given as follows:

1. In the preprocessing, the first step is the Voice Activation Detection (VAD) and segmenting the speech signal accordingly. The VADs obtained for the speech sample of the words 'option' and 'subhash' are given below:

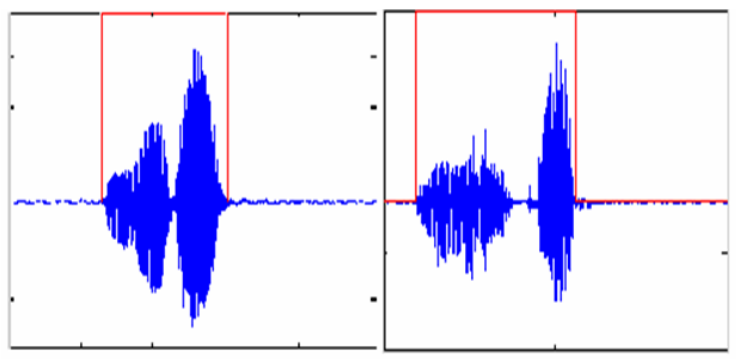

Fig 10: VADs for The Speech Samples

2. The second step of the preprocessing is De-noising. The utterances of the speech sample 'file' with the background noise and after de-noising of the sample are given below:

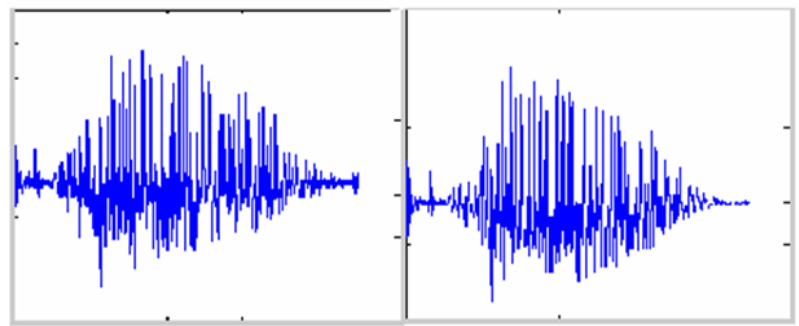

Fig 11: Denoising of the Speech Sample

3. Once the preprocessing is done, the next step is framing and blocking, the frames obtained for the speech sample 'close' of window size $32 \mathrm{~ms}$ with $10 \mathrm{~ms}$ overlapping is given below:

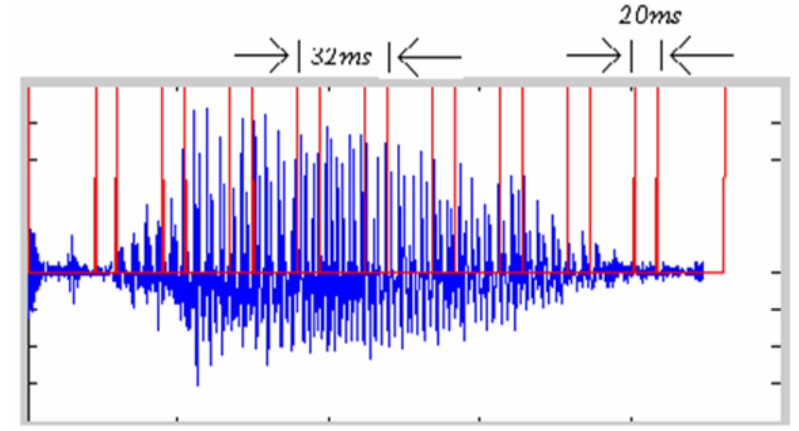

Fig 12: Framing of Speech Sample 'file'

Table 1. Confusion matrix for word classification (in percentage) using Daubechies-8 Wavelet \& 5-level DWT

(90.42\% accuracy)

\begin{tabular}{|c|c|c|c|c|c|c|c|c|c|c|c|c|c|c|c|c|c|c|c|c|}
\hline & $F$ & 桠 & EAl & open & $a_{s z}$ & oit & $\operatorname{cosg}$ & Pate & 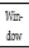 & Deveng & $\begin{array}{l}\text { Sink } \\
\text { मू. }\end{array}$ & 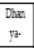 & $\begin{array}{l}\text { Nea } \\
\text { siz }\end{array}$ & Xath & Jeman & Sirat & 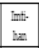 & 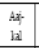 & 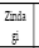 & Slab- \\
\hline Fle & 90.0 & 0 & 0 & 3.3 & 0 & 33 & 0 & 0 & 0 & 0 & 0 & 0 & 0 & 0 & 0 & 33 & 0 & 0 & 0 & 0 \\
\hline Edait & 0 & 833 & 0 & 0 & 33 & 0 & 6.7 & 0 & 0 & 0 & 0 & 0 & 0 & 33 & 0 & 0 & 0 & 33 & 0 & 0 \\
\hline Eajit & 0 & 0 & 933 & 0 & 0 & 0 & 0 & 0 & 0 & 33 & 0 & 0 & 0 & 0 & 0 & 0 & 0 & 0 & 33 & 0 \\
\hline Open & 0 & 0 & 0 & 966 & 0 & 0 & 3.3 & 0 & 0 & 0 & 0 & 0 & 0 & 0 & 0 & 0 & 0 & 0 & 0 & 0 \\
\hline Close & 3.3 & 0 & 0 & 3.3 & 866 & 0 & 0 & 3.3 & 0 & 0 & 0 & 33 & 0 & 0 & 0 & 0 & 0 & 0 & 0 & 0 \\
\hline Cut & 0 & 0 & 0 & 0 & 33 & 833 & 0 & 0 & 0 & 3.3 & 0 & 0 & 0 & 3.3 & 0 & 0 & 0 & 0 & 0 & 6.7 \\
\hline Copf & 0 & 0 & 0 & 6.7 & 0 & 0 & 933 & 0 & 0 & 0 & 0 & 0 & 0 & 0 & 0 & 0 & 0 & 0 & 0 & 0 \\
\hline Passe & 0 & 33 & 0 & 0 & 0 & 0 & 0 & 90.0 & 0 & 33 & 0 & 0 & 0 & 0 & 33 & 0 & 0 & 0 & 0 & 0 \\
\hline Wirdoer & 0 & 0 & 33 & 0 & 0 & 0 & 0 & 0 & 867 & 33 & 0 & 0 & 0 & 0 & 0 & 33 & 0 & 0 & 33 & 0 \\
\hline Detug & 0 & 0 & 33 & 0 & 0 & 3.3 & 0 & 0 & 0 & 933 & 0 & 0 & 0 & 0 & 0 & 0 & 0 & 0 & 0 & 0 \\
\hline Shuldija & 0 & 0 & 0 & 0 & 0 & 0 & 0 & 0 & 0 & 0 & 966 & 0 & 0 & 0 & 0 & 33 & 0 & 0 & 0 & 0 \\
\hline Dhaymad & 0 & 0 & 0 & 33 & 0 & 0 & 0 & 0 & 33 & 0 & 0 & 83.3 & 33 & 0 & 0 & 0 & 0 & 0 & 0 & 6.7 \\
\hline Nanalar & 0 & 0 & 0 & 0 & 0 & 0 & 0 & 0 & 0 & 33 & 0 & 0 & 900 & 0 & 0 & 0 & 33 & 0 & 0 & 3.3 \\
\hline Ritab & 0 & 0 & 0 & 0 & 0 & 0 & 0 & 0 & 0 & 0 & 0 & 33 & 0 & 993 & 0 & 0 & 0 & 0 & 0 & 33 \\
\hline Jerran & 0 & 0 & 0 & 0 & 0 & 0 & 0 & 0 & 0 & 0 & 0 & 0 & 0 & 0 & 96.6 & 0 & 0 & 33 & 0 & 0 \\
\hline Sarase & 0 & 0 & 0 & 0 & 33 & 0 & 0 & 0 & 0 & 0 & 33 & 0 & 0 & 0 & 0 & 993 & 0 & 0 & 0 & 0 \\
\hline Erthan & 0 & 0 & 0 & 0 & 0 & 3.3 & 0 & 0 & 0 & 0 & 0 & 0 & 0 & 6.7 & 33 & 0 & 866 & 0 & 0 & 0 \\
\hline Aspala & 3.3 & 0 & 0 & 0 & 0 & 0 & 3.3 & 0 & 0 & 33 & 0 & 0 & 0 & 0 & 0 & 0 & 0 & 900 & 0 & 0 \\
\hline Zindaggi & 0 & 0 & 0 & 3.3 & 0 & 0 & 0 & 0 & 0 & 67 & 0 & 0 & 6.7 & 0 & 33 & 0 & 0 & 0 & 800 & 0 \\
\hline Shabharh & 0 & 0 & 0 & 0 & 0 & 0 & 0 & 3.3 & 0 & 0 & 0 & 0 & 0 & 0 & 0 & 0 & 0 & 0 & 0 & 967 \\
\hline
\end{tabular}

Table 2. Confusion matrix for word classification (in percentage) using Daubechies-6 Wavelet \& 5-level DWT (84.56\% accuracy)

\begin{tabular}{|c|c|c|c|c|c|c|c|c|c|c|c|c|c|c|c|c|c|c|c|c|}
\hline & $\mathrm{Fla}$ & Ei & Ei & Open & dssa & cit & Copg & Prote & 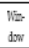 & Deverg & 觜- & $\begin{array}{l}\text { Dax } \\
\text { y }\end{array}$ & $\begin{array}{l}\text { Nima } \\
\text { sitat }\end{array}$ & Xitio & Jera: & Serale & $\begin{array}{ll}=5 \\
b i n\end{array}$ & $\begin{array}{l}A j \\
b i\end{array}$ & $\frac{Z_{\min }}{j}$ & 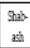 \\
\hline Fle & 83.0 & 0 & 0 & 33 & 0 & 67 & 0 & 0 & 67 & 0 & 0 & 0 & 0 & 0 & 0 & 0 & 0 & 0 & 0 & 0 \\
\hline Eair & 0 & 80.0 & 0 & 0 & 0 & 0 & 6.7 & 33 & 0 & 0 & 0 & 0 & 0 & 0 & 6.7 & 0 & 0 & 33 & 0 & 0 \\
\hline Ejit & 0 & 0 & 866 & 0 & 0 & 0 & 0 & 0 & 33 & 0 & 33 & 0 & 0 & 0 & 0 & 0 & 0 & 0 & 33 & 33 \\
\hline Open & 0 & 0 & 0 & 866 & 0 & 0 & 33 & 0 & 0 & 0 & 0 & 0 & 33 & 0 & 0 & 0 & 0 & 0 & 0 & 67 \\
\hline Close & 3.3 & 0 & 0 & 0 & 866 & 0 & 0 & 3.3 & 0 & 0 & 0 & 0 & 0 & 33 & 0 & 0 & 0 & 33 & 0 & 0 \\
\hline Cut & 33 & 0 & 0 & 0 & 0 & 767 & 0 & 0 & 0 & 0 & 0 & 33 & 0 & 0 & 3.3 & 67 & 0 & 0 & 0 & 67 \\
\hline Copf & 0 & 0 & 33 & 100 & 0 & 0 & 833 & 0 & 0 & 0 & 0 & 0 & 0 & 0 & 0 & 0 & 33 & 0 & 0 & 0 \\
\hline Paste & 0 & 0 & 0 & 0 & 0 & 0 & 0 & 86.6 & 0 & 33 & 0 & 0 & 0 & 6.7 & 3.3 & 0 & 0 & 0 & 0 & 0 \\
\hline Nirdorer & 0 & 0 & 0 & 0 & 0 & 0 & 0 & 0 & 866 & 0 & 0 & 0 & 0 & 6.7 & 0 & 33 & 0 & 0 & 33 & 0 \\
\hline Dewg & 0 & 33 & 0 & 0 & 0 & 33 & 0 & 0 & 0 & 800 & 0 & 0 & 0 & 33 & 67 & 0 & 0 & 0 & 0 & 33 \\
\hline Shlumija & 0 & 0 & 0 & 0 & 0 & 0 & 3.3 & 0 & 0 & 0 & 866 & 0 & 0 & 6.7 & 0 & 0 & 0 & 33 & 0 & 0 \\
\hline Dhaygrad & 0 & 0 & 33 & 0 & 0 & 0 & 0 & 0 & 33 & 0 & 0 & 900 & 0 & 0 & 0 & 0 & 0 & 0 & 0 & 33 \\
\hline Nanaslar & 0 & 0 & 0 & 0 & 33 & 67 & 0 & 0 & 0 & 33 & 0 & 0 & 833 & 0 & 0 & 0 & 0 & 0 & 0 & 33 \\
\hline Kitb & 0 & 33 & 0 & 0 & 0 & 0 & 0 & 0 & 0 & 0 & 0 & 33 & 0 & 866 & 0 & 0 & 33 & 0 & 0 & 33 \\
\hline Jerran & 0 & 0 & 0 & 6.7 & 0 & 0 & 0 & 3.3 & 0 & 0 & 0 & 0 & 0 & 0 & 900 & 0 & 0 & 0 & 0 & 0 \\
\hline Sarase & 0 & 0 & 0 & 0 & 0 & 33 & 0 & 0 & 0 & 0 & 3.3 & 0 & 0 & 0 & 0 & 866 & 0 & 0 & 0 & 67 \\
\hline Erthan & 0 & 0 & 0 & 0 & 0 & 67 & 0 & 0 & 3.3 & 0 & 0 & 0 & 0 & 0 & 67 & 33 & 766 & 0 & 33 & 0 \\
\hline Askal & 3.3 & 0 & 0 & 0 & 0 & 0 & 0 & 0 & 0 & 33 & 0 & 33 & 0 & 0 & 0 & 0 & 0 & 900 & 0 & 0 \\
\hline Zindagi & 0 & 0 & 0 & 33 & 0 & 0 & 0 & 0 & 0 & 67 & 0 & 0 & 0 & 0 & 0 & 0 & 0 & 0 & 900 & 0 \\
\hline Stabahh & 0 & 0 & 0 & 0 & 33 & 0 & 6.7 & 0 & 0 & 0 & 0 & 0 & 0 & 6.7 & 0 & 0 & 0 & 0 & 0 & 833 \\
\hline
\end{tabular}




\section{CONCLUSION}

The features obtained by using the wavelet transform shows higher recognition rates if the features are extracted properly. Wavelets proved to have both strengths and weaknesses for speech feature identification. In general, wavelets are able to distinguish between different properties high frequency low amplitude spectral components and low frequency large amplitude spectral components Also, neural network classifier improves the recognition performance significantly. The result shows that the wavelet transform can be effectively used for the extraction of features for speaker independent word recognition. Higher recognition performance can be achieved by using more complex classification techniques.

\section{FUTURE WORK}

The exact details of feature calculation for MFCC have been explored extensively in the literature. On the other hand, wavelet based features have appeared relatively recently. Further improvements in classification accuracy can be expected with more careful experimentation with the exact details of the parameters and for other wavelet families. Another interesting direction is combining features from different analysis techniques to improve classification accuracy.

\section{REFERENCES}

[1] B.T. Tan, M. Fu, A. Spray, P. Dermody, "The use of wavelet transform for phoneme recognition," Proceedings of the $4^{\text {th }}$ International Conference of Spoken Language Processing Philadelphia, Vol. 4, USA, October 1996, pp.2431-2434.

[2] S. G. Mallat, "A theory for multiresolution signal decomposition: the wavelet representation," IEEE transactions on Pattern Analysis Machine Intelligence, Vol. 11 1989, pp.674-693.
[3] Oliver Siohan and Chin-Hui Lee "Iterative Noise and Channel Estimation under the Stochastic Matching Algorithm Framework" IEEE Signal Processing, Processing Letters, Vol. 4, No. 11, Nov 1997.

[4] M. Misiti, Y. Misiti, G. Oppenheim and J. Poggi, Matlab Wavelet Tool Box, The Math Works Inc.,2000 Page: 795.

[5] George Tzanetakis, Georg Essl, Perry Cook, "Audio Analysis using the Discrete Wavelet Transform" Organized sound, Vol. 4(3), 2000.

[6] L. Barbier, G. Chollet, "Robust speech parameters extraction for word recognition in noise using neural networks," IEEE International Conference on Acoustics, Speech, and Signal Processing, Pages: 145-148, May 1991.

[7] X. Huang, "Speaker normalization for speech recognition", IEEE International Conference on Acoustics, Speech, and Signal Processing, 1:465-468, March 1992.

[8] S. Tamura, A Waibel, "Noise reduction using connectionist models." IEEE International Conference on Acoustics, Speech, and Signal Processing, 1:553-556, April 1988

[9] S. Young, "A review of large vocabulary continuesspeech recognition," Proc. IEEE Sig. Processing. Mag. (September) (1996) 45-57.

[10] N. Desmukh, A. Ganapathiraju, J. Picone, "Hierarchical search for large vocabulary conversational speech recognition - working toward a solution to the decoding problem," IEEE Sig, Process Mag. (September) (1999) 84-107. 